\title{
Modeling of long length and complex shaped chain and planed conveyor noise forming
}

\author{
Mariya Balinskaya ${ }^{1,{ }^{*}}$, Besarion Meskhi ${ }^{1}$ and Igor Khozyaev $^{1}$ \\ ${ }^{1}$ Don State Technical University, 1, Gagarin sq., 344003, Rostov-on-Don, Russia
}

\begin{abstract}
The article presents an analysis of the chain-slat conveyor in terms of noise generation. It is shown that, due to the volumetric layout and long length, it is one of the main sources of noise generation. Based on these studies, a theoretical model of its noise generation is developed. The model has been tested experimentally. The coincidence of the calculation of the results and experimental data is quite acceptable. This gives reason to talk about the possibility of using the model for calculating the noise of the conveyor of other schemes.
\end{abstract}

\section{Introduction}

In the food industry, various conveyors are widely used to move products and goods. They are continuous transport machines. By design, they are divided into two large groups: conveyors with a flexible traction body and without a flexible one. At food enterprises use only the first group. The most widespread are chain plate conveyors. They serve to move goods in horizontal, inclined and vertical directions.

An endless, vertically closed chassis of a conveyor consists of a load-bearing plate deck attached to a traction element, usually in the form of one or two chains. The transported cargo is moved by the upper branch of the conveyor; the lower branch is single.[1]

The chassis bends around - tail - tension and head - traction (drive) sprockets; in the middle part, it is supported by guide rails or stationary rollers attached to the frame of the bed. Traction sprockets receive rotation from a drive mechanism consisting of an electric motor, gearbox or variator. Chain-plate conveyors can have a large length, deployed in space. Figure 1 shows the scheme of operation of the chain-plate conveyor of the Rostov Champagne Wines Plant (RKSV), and Figure 2 shows the drive of its various branches.[2]

\footnotetext{
*Correspondingauthor: mbalinskaya91@gmail.com
} 


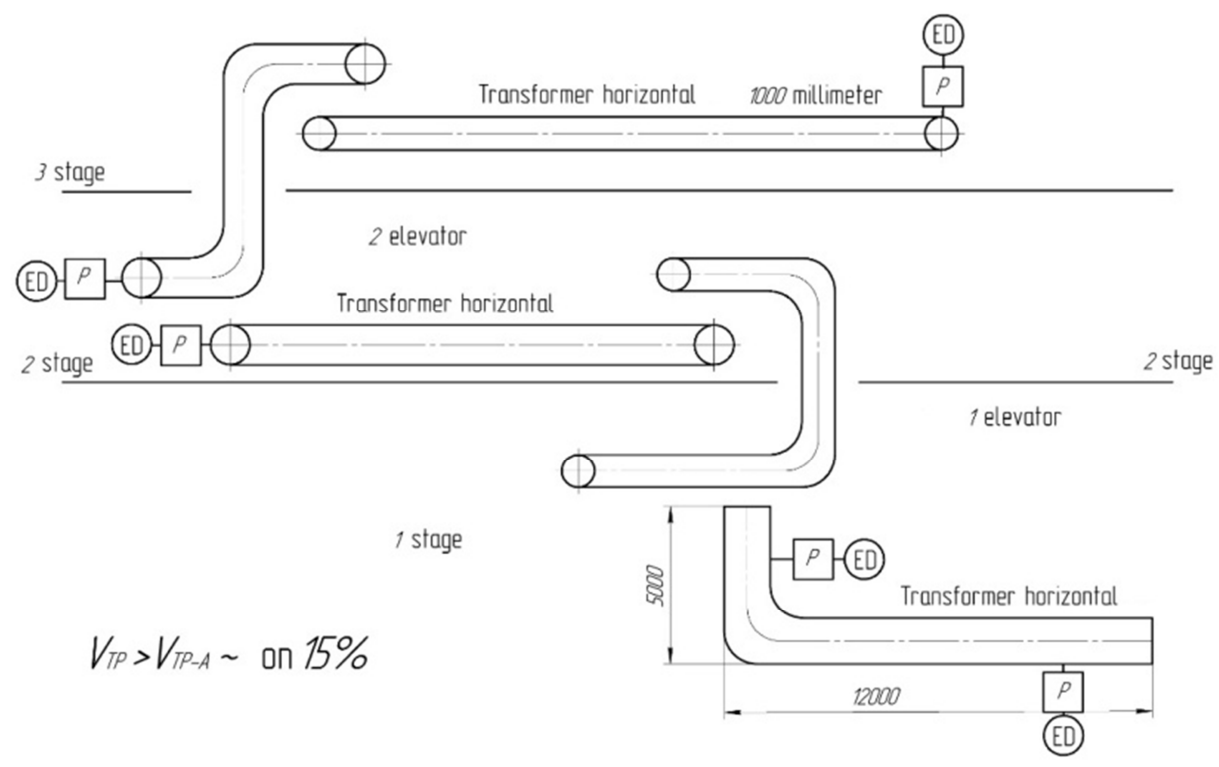

Fig. 1. Scheme for calculating the sound pressure of chain-plate conveyors RKSV.

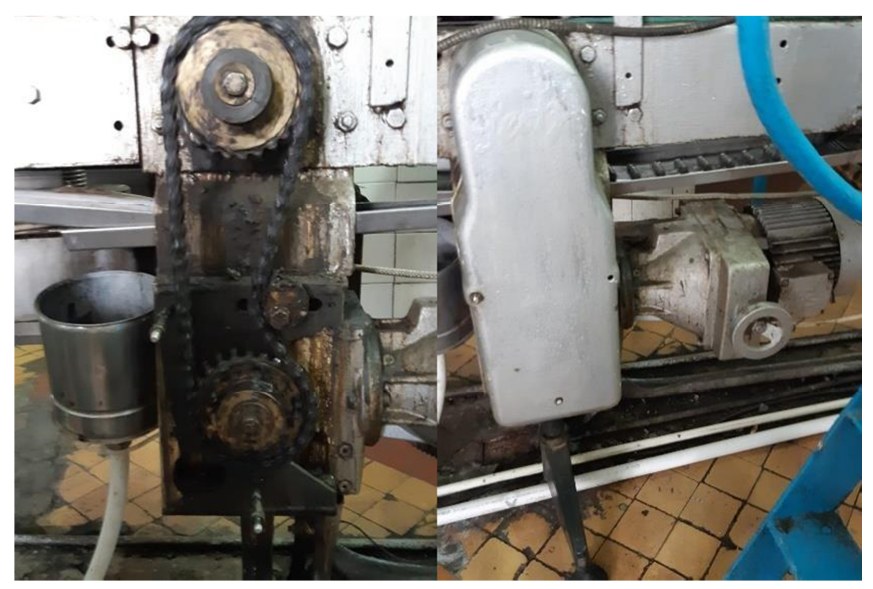

Fig. 2. General view of the conveyor drives: a - with gear; $b$ - with a variator.

In order to avoid complex synchronization schemes, these conveyors are idling constantly and, if necessary, are loaded with empty bottles, which are then filled with wine on the 3rd floor, sealed, formed into lots and packed in boxes. In connection with this mode of operation, the conveyor is one of the main sources of noise, which together with other sources creates a common noise field, significantly exceeding standard values [3-5].

There are practically no works on assessing the noise generation of plate banners, and especially theoretical ones, which means that it is difficult to predict the influence of their operating modes on the BZD of enterprises where such machines are used. Therefore, based on the foregoing, it can be noted that the development of a theoretical model of noise generation during plate-chain conveyor operations is an urgent task.

\section{Materials and Methods}


The conveyor has a constant moment in its corresponding sections along the $\mathrm{z}$ coordinate (take the direction of the $\mathrm{OZ}$ axis along the conveyor), inertia moments in direction of the coordinate axes OX and OY are different. Therefore, as a calculation model, a rod (bank) was adopted, which receives force disturbances from the weight of the moved tare and the conveyor drive (Fig.3) [6].

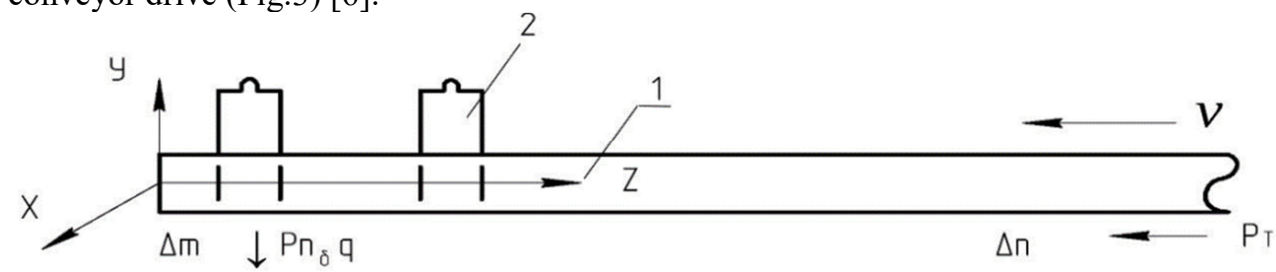

Fig. 3. The design scheme of the conveyor.

Where : 1 - conveyor housing; 2 - container; $\mathrm{m}_{\delta}$ - bottle weight; $\mathrm{g}=1,4 \frac{\mathrm{m}}{\mathrm{sec}^{2}}$ gravity acceleration; $\mathrm{P}_{\mathrm{T}}-$ drive pull, $\mathrm{H} ; \vartheta$ - conveyor speed, $\frac{m}{\mathrm{sec}}$.

Since the conveyor is a system with a distributed mass, the differential equations of oscillations have the form: [7-8]

$$
E Y_{Y} \frac{\partial^{4} y}{\partial z^{4}}+m_{0} \frac{\partial^{2} y}{d t^{2}}=P(t) \delta\left(Z-Z_{0}\right)
$$

where $\delta\left(Z-Z_{0}\right)$ - function taking into account the coordinates of the application of force; $\mathrm{Y}_{\mathrm{y}}-$ moment of inertia, $\mathrm{m}^{4} ; \mathrm{E}$ - elastic modulus, $\mathrm{Pa} ; \mathrm{m}_{0}-$ distributed mass, $\mathrm{kg} / \mathrm{m} ; \mathrm{P}(\mathrm{t})$ force measurement amplitude, $\mathrm{m}$.

Given that the tare filling weight is the same, and also using the method of separation of variables, the following differential equations of conveyor vibrations are obtained: [9-10] Container without liquid

$$
\begin{aligned}
& \frac{d^{2} y_{1}}{d t}+2,6 * 10^{9} \frac{Y_{y}}{F_{T}}\left(\frac{k}{l}\right)^{4} y_{1} \\
& =\frac{5\left(D^{2}-d^{2}\right) l_{\delta} k_{\delta}}{F_{T} l} \sin \frac{\pi k \vartheta}{l} t \\
& +\frac{6,5 * 10^{-4} P_{T}}{F_{T}}\left[\cos \left(\frac{\pi k \vartheta}{l}-\frac{\pi k Z^{x}}{30}\right) t\right] \\
& \frac{d^{2} x_{1}}{d t^{2}}+2,6 * 10^{9} \frac{Y_{y}}{F_{T}}\left(\frac{k}{l}\right)^{4} y_{2} \\
& =\frac{5\left(D^{2}-d^{2}\right) l_{\delta} k_{\delta}}{F_{T} l} \sin \frac{\pi k \vartheta}{l} t \\
& +\frac{6,5 * 10^{-4} P_{T}}{F_{T}}\left[\cos \left(\frac{\pi k \vartheta}{l}-\frac{\pi k Z^{x}}{30}\right) t-\cos \left(\frac{\pi k \vartheta}{l}-\frac{\pi k Z^{x}}{30}\right) t\right]
\end{aligned}
$$

whereV - conveyor speed, $\mathrm{m} / \mathrm{sec}$.

Liquid container 


$$
\begin{gathered}
\frac{d^{2} y_{2}}{d t^{2}}+2,6 * 10^{9} \frac{Y_{y}}{F_{T}}\left(\frac{k}{l}\right)^{4} y_{2} \\
=\frac{1,6 * 10^{-3} l_{\delta}\left[2500\left(D^{2}-d^{2}\right)+\rho_{\text {ж }} d^{2}\right]}{F_{T} l} \sin \frac{\pi k \vartheta}{l} t \\
+\frac{6,5 * 10^{-4} P_{T}}{F_{T}}\left[\cos \left(\frac{\pi k \vartheta}{l}-\frac{\pi k Z^{x}}{30}\right) t-\cos \left(\frac{\pi k \vartheta}{l}-\frac{\pi k Z^{x}}{30}\right) t\right]
\end{gathered}
$$

$$
\begin{aligned}
\frac{d^{2} x_{2}}{d t^{2}}+2,6 * 109 & \frac{Y_{x}}{F_{T}}\left(\frac{k}{l}\right)^{4} x_{2} \\
& =\frac{1,6 * 10^{-3} l_{\delta}\left[2500\left(D^{2}-d^{2}\right)+\rho_{\text {ж }} d^{2}\right]}{F_{T} l} \sin \frac{\pi k \vartheta}{l} t \\
& +\frac{6,5 * 10^{-4} P_{T}}{F_{T}}\left[\cos \left(\frac{\pi k \vartheta}{l}-\frac{\pi k Z^{x}}{30}\right) t-\cos \left(\frac{\pi k \vartheta}{l}-\frac{\pi k Z^{x}}{30}\right) t\right]
\end{aligned}
$$

where 1 - conveyor length, $\mathrm{m} ; \mathrm{F}_{\mathrm{T}}$ - conveyor cross-sectional area, $\mathrm{m}^{2}$.

Solutions of these equations with respect to the modulus of oscillation velocities are obtained as follows:

$$
\begin{aligned}
\left|\vartheta_{k_{1}}\right|=\left|\frac{\partial y_{1}}{\partial t}\right|= & \mid \frac{16\left(D^{2}-d^{2}\right) l_{\delta} k_{\delta} \vartheta}{F_{T}} \sum_{k=1}^{k^{x}} \frac{k \cos \frac{\pi k \vartheta}{l}}{2,6 * 10^{9} \frac{Y_{x}}{F_{T}}\left(\frac{k}{l}\right)^{4}-\left(\frac{\pi k \vartheta}{l}\right)^{2}} \\
& +\frac{6,5 * 10^{-4} P_{T}}{F_{T}} \sum_{k=1}^{k^{x}}-\frac{\left(\frac{\pi k \vartheta}{l}-\frac{\pi k Z^{x}}{30}\right) \sin \left(\frac{\pi k \vartheta}{l}-\frac{\pi k Z^{x}}{30}\right) t}{2,6 * 10^{9} \frac{Y_{y}}{F_{T}}\left(\frac{k}{l}\right)^{4}-\left(\frac{\pi k \vartheta}{l}-\frac{\pi k Z^{x}}{30}\right)^{2}} \\
& +\frac{\left(\frac{\pi k \vartheta}{l}-\frac{\pi k Z^{x}}{30}\right) \sin \left(\frac{\pi k \vartheta}{1}-\frac{\pi k Z^{x}}{30}\right) t}{2,6 * 10^{9} \frac{Y_{y}}{F_{T}}\left(\frac{k}{l}\right)^{4}-\left(\frac{\pi k \vartheta}{l}-\frac{\pi k Z^{x}}{30}\right)^{2} \mid} \\
\left|\vartheta_{k_{2}}\right|=\left|\frac{\partial x_{1} \mid}{\partial t}\right|=\mid & \mid \frac{16\left(D^{2}-d^{2}\right) l_{\delta} k_{\delta} \vartheta}{F_{T}} \sum_{k=1}^{k^{x}} \frac{k \cos \frac{\pi k \vartheta}{l}}{2,6 *\left(10^{9} \frac{Y_{x}}{F_{T}}\left(\frac{k}{l}\right)^{4}-\left(\frac{\pi k \vartheta}{1}\right)^{2}\right.} \\
& +\frac{6,5 * 10^{-4} P_{T}}{F_{T}} \sum_{k=1}^{k^{x}}-\frac{\left(\frac{\pi k \vartheta}{l}-\frac{\pi k Z^{x}}{30}\right) \sin \left(\frac{\pi k \vartheta}{l}-\frac{\pi k Z^{x}}{30}\right) t}{2,6 * 10^{9} \frac{Y_{y}}{F_{T}}\left(\frac{k}{l}\right)^{4}-\left(\frac{\pi k \vartheta}{l}-\frac{\pi k Z^{x}}{30}\right)^{2}} \\
& +\frac{\left(\frac{\pi k \vartheta}{l}-\frac{\pi k Z^{x}}{30}\right) \sin \left(\frac{\pi k \vartheta}{1}-\frac{\pi k Z^{x}}{30}\right) t}{2,6 * 10^{9} \frac{Y_{y}}{F_{T}}\left(\frac{k}{l}\right)^{4}-\left(\frac{\pi k \vartheta}{l}-\frac{\pi k Z^{x}}{30}\right)^{2} \mid}
\end{aligned}
$$




$$
\begin{aligned}
& \left|\vartheta_{k_{3}}\right|=\left|\frac{\partial y_{2}}{\partial t}\right|=\mid \frac{5 * 10^{-3} l_{\delta}\left[2500\left(D^{2}-d^{2}\right)+2500 d^{2}\right]}{F_{T} l^{2}} \\
& * \sum \frac{k \cos \frac{\pi k \vartheta}{l} t}{2,6 * 10^{9} \frac{Y_{y}}{F_{T}}\left(\frac{\pi k}{l}\right)^{4}-\left(\frac{\pi k \vartheta}{l}\right)^{2}} \\
& +\frac{6,5 * 10^{-4} P_{T}}{F_{T}} \sum_{k=1}^{k^{x}}-\frac{\left(\frac{\pi k \vartheta}{l}-\frac{\pi k Z^{x}}{30}\right) \sin \left(\frac{\pi k \vartheta}{1}-\frac{\pi k Z^{x}}{30}\right) t}{2,6 * 10^{9} \frac{Y_{y}}{F_{T}}\left(\frac{k}{l}\right)^{4}-\left(\frac{\pi k \vartheta}{l}-\frac{\pi k Z^{x}}{30}\right)^{2}} \\
& +\frac{\left(\frac{\pi k \vartheta}{l}-\frac{\pi k Z^{x}}{30}\right) \sin \left(\frac{\pi k \vartheta}{1}-\frac{\pi k Z^{x}}{30}\right) t}{2,6 * 10^{9} \frac{Y_{y}}{F_{T}}\left(\frac{\pi k}{l}\right)^{4}-\left(\frac{\pi k \vartheta}{1}-\frac{\pi k Z^{x}}{30}\right)^{2}} \mid \\
& \left|\vartheta_{k_{4}}\right|=\left|\frac{\partial x_{2}}{\partial t}\right|=\mid \frac{5 * 10^{-3} l_{\delta}\left[2500\left(D^{2}-d^{2}\right)+2500 d^{2}\right]}{F_{T} l^{2}} \\
& * \sum \frac{k \cos \frac{\pi k \vartheta}{l} t}{2,6 * 10^{9} \frac{Y_{y}}{F_{T}}\left(\frac{\pi k}{l}\right)^{4}-\left(\frac{\pi k \vartheta}{l}\right)^{2}} \\
& +\frac{6,5 * 10^{-4} P_{T}}{F_{T}} \sum_{k=1}^{k^{x}}-\frac{\left(\frac{\pi k \vartheta}{l}-\frac{\pi k Z^{x}}{30}\right) \sin \left(\frac{\pi k \vartheta}{1}-\frac{\pi k Z^{x}}{30}\right) t}{2,6 * 10^{9} \frac{Y_{y}}{F_{T}}\left(\frac{k}{l}\right)^{4}-\left(\frac{\pi k \vartheta}{l}-\frac{\pi k Z^{x}}{30}\right)^{2}} \\
& +\frac{\left(\frac{\pi k \vartheta}{l}-\frac{\pi k Z^{x}}{30}\right) \sin \left(\frac{\pi k \vartheta}{1}-\frac{\pi k Z^{x}}{30}\right) t}{2,6 * 10^{9} \frac{Y_{y}}{F_{T}}\left(\frac{\pi k}{l}\right)^{4}-\left(\frac{\pi k \vartheta}{1}-\frac{\pi k Z^{x}}{30}\right)^{2}} \mid
\end{aligned}
$$

where $\mathrm{n}$ - sprocket speed; $\mathrm{z}$ - number of teeth; $\mathrm{k}$ - coefficient determining the corresponding oscillation frequencies of the conveyor; $\mathrm{D}$ и $\mathrm{d}$ - outer and inner diameters of containers, $\mathrm{m} ; \mathrm{l}_{\delta}$ - tare height, $\mathrm{m} ; \mathrm{n}$ - number of containers; $\mathrm{P}_{\mathrm{t}}$ и $\mathrm{P}_{\text {ж}}$ - density of containers and liquids(respectively), $\frac{\mathrm{kg}}{\mathrm{m}^{3}} ; \mathrm{k}$ - the number of natural frequencies of the conveyor in the normalized frequency range.[11-13]

$$
f_{k}=\frac{\pi k^{2}}{2 l^{2}} \sqrt{\frac{E J}{\rho F}}=8 * 10^{3} \frac{Y}{l^{2}}\left(\frac{Y}{F_{T}}\right)^{0,5},
$$

Given that according to the normalized frequency range, $\mathrm{f}_{\mathrm{k}_{\max }}=11200109$, we get:

To calculate the acoustic characteristics, the maximum value of the rms vibration velocity was used:

$$
\vartheta_{k \max }=\sqrt{\left(\vartheta_{k 1}+\vartheta_{k 3}\right)^{2}+\left(\vartheta_{k 2}+\vartheta_{k 4}\right)^{2}}
$$


Based on the obtained values of the oscillation velocity, the main sound pressure levels are determined $\mathrm{lp}$ and power $\mathrm{l}_{\mathrm{w}}(1,3)$ :

$$
\begin{aligned}
& l_{p}=20 \lg \frac{\left(f_{k} S \prime l\right)^{0,5} \vartheta_{k}}{2 * 10^{-5} Z}, \\
& l_{w}=10 \lg \frac{0,659 \vartheta_{k} l f_{k} S^{\prime}}{10^{-12}},
\end{aligned}
$$

where $\mathrm{S}$ - conveyor surface area, $\mathrm{m}^{2} ; \mathrm{Z}$ - distance from source to design point, $\mathrm{m}$. Sound power levels or sound pressure created by the simultaneous study of the sound energy of all sources is determined by energy summation:

$$
l_{z}=10 \lg 10^{0,1 L_{t}}+k_{\delta} * 10^{0,1 L_{\delta}},
$$

where $\mathrm{L}_{\mathrm{t}}-$ conveyor sound power (sound pressure) levels, $\mathrm{dB} ; \mathrm{L}_{\delta}-$ sound power levels (sound pressure) of bottles, $\mathrm{dB}$.

The noise levels generated in the production room on the basis of these works $(5,6,7)$ were obtained:

$$
l=l_{z}+10 \lg \left(\frac{0,12}{z^{2}}+\frac{1-1,3 a}{a S_{n}}\right)
$$

where $\mathrm{S}_{\mathrm{n}}$ - floor space, $\mathrm{m}^{2} ; \alpha$-average sound absorption coefficient. The calculation of sound pressure levels is performed according to the algorithm(Fig. 4):[14-17]

Definition of arrays of natural frequencies of oscillations of each source

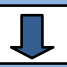

The allocation of octave bands into which the oscillation frequencies of noise sources fall

Noise Source Equations

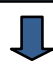

Determination of vibration rates

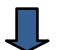

Energy summation of the levels of octave sources in the respective octaves

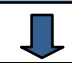

Definitions of sound power equations

Fig. 4. General algorithm for calculating noise equations using equations (10) - (11).

Based on equation (10) - (11), a program for the PC was developed and that the sound pressure levels of the chain-plate conveyor shown in Fig. 2 were calculated from Fig. 1. The adequacy of the developed models was verified by comparing the calculated and experimental results. In Fig. 4 shows the recording of noise during the operation of the conveyor.[18] 


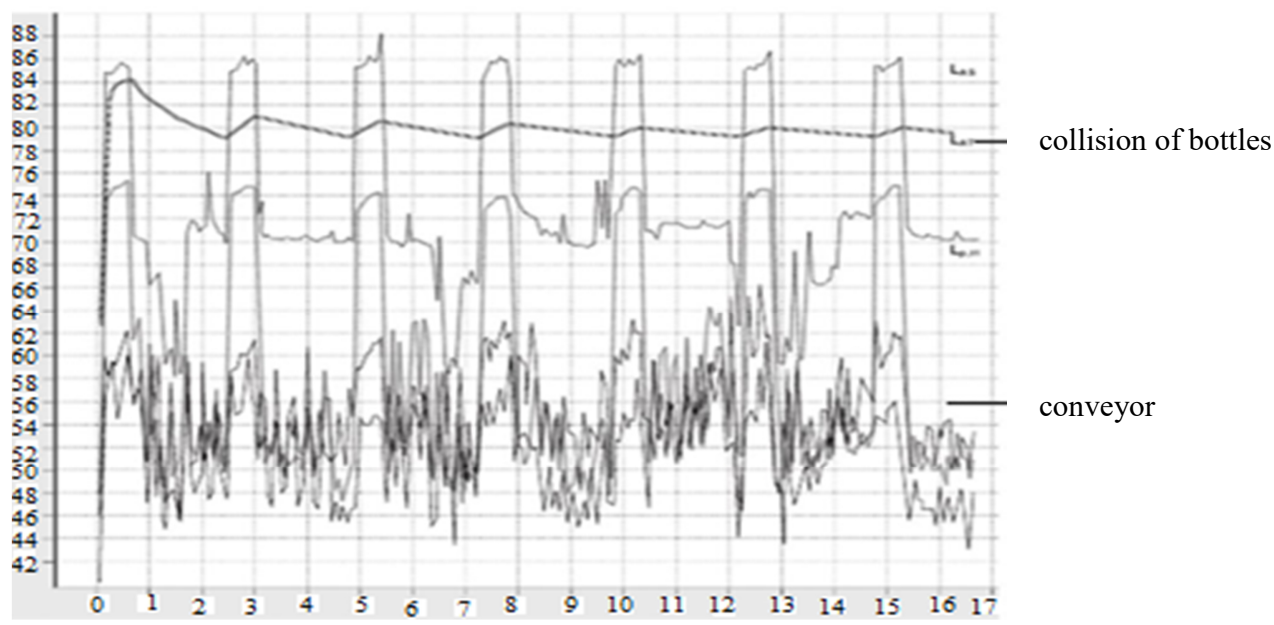

Fig. 5. Sound level $L_{A S}, L_{A T}$ and sound pressure $L_{p, 1100}, L_{p, 180}, L_{p, 125}(1 / 3$ octave 100,80 and $125 \mathrm{~Hz})$ conveyor of empty bottles.

Analyzing the spectra, one can notice that from time to time a tonal component of 100 $\mathrm{Hz}$ appears in the 1/3-octave spectrum (Fig. 2).[19-20]

In the time history (Fig. 1), this tonal component is shown by a curveLpr.100. It is clearly seen that it appears every time at the beginning of the working cycle, and the SPL in adjacent bands $(80 \mathrm{~Hz}$ and $125 \mathrm{~Hz}$ ) is much lower.

Statistical processing of the results showed that, starting from $1000 \mathrm{~Hz}$, the theoretical value by $2-2.5$ oak exceeds the experimental value, and at a frequency of up to $500 \mathrm{~Hz}$ the experimental values exceed the calculated values by the same amount of 2-2.5 dB.

\section{Conclusion}

1. It has been experimentally confirmed that a chain-slat conveyor of complex volumetric shape is a strong noise-generating element of transport lines.

2. The developed theoretical model of noise formation of the chain-slat conveyor is adequate and allows you to calculate sound pressure levels at different frequencies.

3. Knowledge of the noise characteristics of such a device (theoretical and experimental) allows already at the stage of the conveyor design to develop noise protection measures.

\section{References}

1. E.V. Rachkov, Continuous Transport Machines (Altair-MGAVT, Moscow, 2014)

2. Y.P. Shcheviev, Fundamentals of Physical Acoustics (Doe, St. Petersburg, 2017)

3. V. . Butyrin, V.N. Maksimenko, L.V. Pavshok, B.S. Reznikov, Strength calculations and optimal weight design multilayered shell products from composites if exposed to loads (Applied mechanics and technical physics, 2014)

4. S.V. Popkov, Vibroacoustic measurements (Publishing House of St. Petersburg Electrotechnical University "LETI", St. Petersburg, 2018)

5. V.N. Bobylev, D.V. Monich, V.A. Tishkov, P.A. Grebnev, Reserves for improving the sound insulation of single-layer enclosing constructions (NNGASU, N. Novgorod, 2014) 
6. B.C. Meskhi, K.O. Kobzev, S.F. Podust, I.M. Chukarina, S.A. Shamshura,Design of noise reduction systems for equipment with a high-frequency pulsed nature of the process: textbook. allowance (DGTU, Rostov-on-Don, 2019)

7. B.C. Meskhi, K.O. Kobzev, V.G. Lebedenko, A.V. Brain, I.M. Chukarin, Designing systems to increase the efficiency of processing parts by shots provided that noise and vibration are reduced at the operator's workplace: textbook. allowance (DSTU, Rostov-on-Don, 2019)

8. S.K. Dargar, J.K. Srivastava, Pruning Technique of ANN for Proficient Active Noise Cancellation: An Experimental study (LAP LAMBERT Academic Publishing, 2016)

9. A. Thumann, Fundamentals of noise control engineering (Prentice-Hall, 2016)

10. D.A. Bies, C. Hansen, C. Howard, Engineering Noise Control (University of Adelaide, Australia, 2017)

11. R.J. Peters, Acoustics and Noise Control (Routledge, 2013) 